\title{
Transoral robotic surgery: development and challenges
}

This article was published in the following Dove Press journal:

Robotic Surgery: Research and Reviews

12 January 2015

Number of times this article has been viewed

\section{Raymond K Tsang}

Department of Surgery, University of Hong Kong, Hong Kong
Correspondence: Raymond K Tsang Department of Surgery, University of Hong Kong, Queen Mary Hospital, 102 Pokfulam Road, Hong Kong

Tel +852 22554394

Fax +85228193780

Email rkytsang@hku.hk
Abstract: The da Vinci Surgical robot has been marketed since 1999; it was originally designed for aiding complex laparoscopic operations and cardiac operations. By the mid-2000s, otolaryngologists had been attempting to deploy the surgical robot for endoscopic minimally invasive operations in the upper aerodigestive tract. The development went through a mannequin model, canine model, and cadaver model and finally cumulated to human Phase I trial. The operation was dubbed transoral robotic surgery (TORS). Subsequently, multicenter trials in the United States had proved the safety and oncological efficacy of using the surgical robot for resection of early cancers in the upper aerodigestive tract. In 2009, the US Food and Drug Administration granted license for the da Vinci surgical robot to be used for resection of benign tumors and early cancers in the oropharynx, larynx, and hypopharynx. TORS has also developed for surgery in the anterior skull base and nasopharynx, as well as in setting of flaps for reconstruction in the upper aerodigestive tract. This article will review the development of TORS, the current limitations, and future developments.

Keywords: head and neck surgery, minimally invasive surgery, computer aided surgery, endoscopic surgery

\section{Introduction}

Technology has been the driving force of medical advances. The introduction of the charged coupled device that was small enough to adapt to a rigid endoscopy enabled images of the internal organs inside the abdomen viewed by a laparoscope to be displayed on a television monitor screen. This led to the development of the first laparoscopic cholecystectomy in 1998 and endoscopic surgery has become the standard of care for a variety of diseases in many subspecialties.

Telemanipulation is a technology that enabled the movement of a remote effector (robot) to copy the movement of the operator. It is the basis of robotic arms. Raymond Geortz developed the first robotic arm with master and slave configuration for handling of radioactive substance in the Argonne National Laboratory in Chicago, USA, in 1951.

The combination of the two technologies of remote visualization and telemanipulation formed the core technology of the da Vinci surgical robot. The da Vinci surgical robot was developed by Intuitive Surgical Inc. (Sunnyvale, CA, USA). The company was founded in 1995 and the first clinical application was performed in March 1997. The operation was a laparoscopic cholecystectomy. ${ }^{1}$ The da Vinci surgical robot was marketed in 1999 and obtained approval for clinical use in laparoscopic surgery by the Food and Drug Administration of USA (FDA) in 2000. 
The da Vinci surgical robot consisted of a control console and a patient cart. The patient cart had three and later four robotic arms that control the instruments inserted into the patient, and the patient cart is placed near the patient at the side of the operating table. One arm is reserved for controlling the endoscope for visualization. The surgeon sits on the control console, which is placed at a distance from the patient but inside the operation room. The surgeon would be able to visualize the view of the endoscope on the control console and control the movements of the robotic arms.

The da Vinci surgical robot is designed to be an augmentation of conventional laparoscopic surgery. In conventional laparoscopic surgery, the distal ends of the instruments are usually not articulated. Even if they are articulated, simultaneous control of all the joints would be difficult. Therefore, in conventional laparoscopic surgery, some degree of movement will need to be sacrificed. The major advantage of the da Vinci surgical robot is the incorporation of the EndoWrist ${ }^{\circledR}$, which allowed 7 degrees of movement and 540 degrees of arm rotation. The distal articulation of the wristed instrument has a larger range of motion than a human wrist and is miniaturized to allow placement in tight spaces.

The other advantages of the da Vinci surgical robot include three-dimensional images, motion scaling for precise movements of the instruments, tremor filtration, and lack of fatigue. ${ }^{2}$ Remote surgery and remote proctoring are also added advantages in certain locations in the world where expertise may not be directly accessible.

Two years after the da Vinci surgical robot had been marketed, substantial experience was gained in abdominal surgery. ${ }^{2}$ After a decade of development, the use of the da Vinci surgical robot in minimally invasive surgery has shown advantages in the field of urology, ${ }^{3}$ gynecology, ${ }^{4}$ and colorectal surgery. ${ }^{5}$

The da Vinci surgical robot is not designed primarily for head and neck surgery, but head and neck surgeons have shown interest in adapting the da Vinci surgical robot to perform minimally invasive surgery or remote-access surgery. The use of the da Vinci surgical robot in the head and neck area can be divided into: 1) minimally invasive surgery where the surgical robot is used to perform surgical operations inside the upper aerodigestive tract without transgressing the normal tissue of the head and neck region; and 2) remote access surgery to perform surgery in the neck through incisions placed away from the organ in cosmetically acceptable regions of the neck.

The present article is a review of the current state of robot surgery in the head and neck region, mainly focusing on the application of the da Vinci surgical robot. PubMed was the primary database consulted in preparing this review and all the literature reviewed was in English. The review is not conducted as an exhaustive literature search like a meta-analysis, but is intended to provide an overview of the current state of robotic surgery in the head and neck region. Table 1 lists the robotic procedures mentioned in the review.

\section{Application of the da Vinci surgical robot in upper aerodigestive tract}

McLeod and Melder performed the first application of the da Vinci surgical robot in endolaryngeal surgery in $2002 .{ }^{6}$ The robot was used to resect a vallecular cyst. The case was a proof of concept, opening up the use of the robot in the field of head and neck surgery.

Subsequently, extensive research on application of the da Vinci surgical robot in performing endoscopic surgery in the oropharynx, larynx, and hypopharynx was performed by the University of Pennsylvania group. The study started on an airway mannequin, ${ }^{7}$ progressing to cadaveric dissections, ${ }^{8-10}$ and later to a canine model..$^{11,12}$ After the success in the preclinical phase of the study, the group embarked on Phase I clinical trials in 2006 with case series on resection of base of tongue cancers, ${ }^{8}$ tonsillar cancers, ${ }^{13}$ and supraglottic cancers. ${ }^{14}$ The FDA approved the use of the da Vinci surgical robot for endoscopic resection of early T-stage (T1-T2) head and neck cancers in 2009. The operation was subsequently named "transoral robotic surgery" (TORS). Subsequently, other centers applied TORS for resection of early cancers in the hypopharynx. ${ }^{15}$

Several centers subsequently showed that TORS resection of early stage cancer of the upper aerodigestive tract was technically feasible and TORS resection was able to obtain clear resection margins. ${ }^{13,16-18}$ The quoted advantage of TORS compared to other endoscopic resection methods like endoscopic laser surgery included better access to tumors. The angled endoscope and EndoWrist ${ }^{\circledR}$ allowed access to tumors outside the line of sight, which would not be accessible with traditional endoscopic instruments and $\mathrm{CO}_{2}$ laser. After an initial learning phase, the operating time including robot setup time was comparable to traditional endoscopic surgery. The postoperative recovery, including time to return to oral diet and hospital stay, was also comparable. Figures 1-5 illustrate a typical case of robotic tonsillectomy for $\mathrm{T} 1$ cancer of the tonsil.

TORS and laser surgery are not mutually exclusive technologies. Several centers had adapted the use of fiber laser delivery with the da Vinci robot in resection of oropharyngeal, laryngeal, and hypopharyngeal lesions. The advantages of the laser included precise incision, minimal 


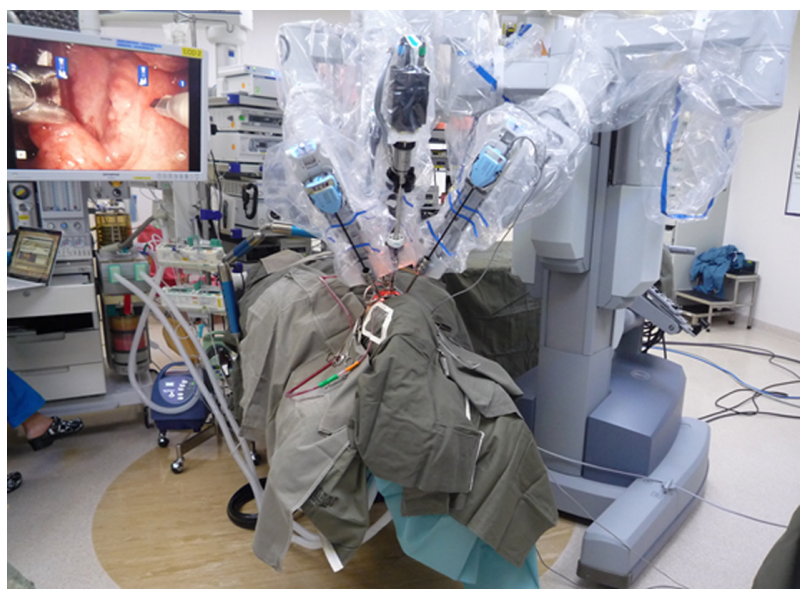

Figure I View of the setup of the patient and the da Vinci surgical robot for robotic radical tonsillectomy.

adjacent tissue damage, and excellent tissue hemostasis. ${ }^{19-22}$ The EndoWrist ${ }^{\circledR}$ and angled telescope of the da Vinci surgical robot allow delivery of laser to areas previously not able to be reached by traditional delivery methods like transoral laser microscopic surgery.

Short-term oncological results of TORS have been shown to be comparable to other treatment methods like radical resection or concurrent chemoradiotherapy in several centers. Most of the short-term oncological outcomes were reports on the results of treating oropharyngeal cancers. ${ }^{23-31}$ Unfortunately, a high proportion of the patients in the above cohorts received postoperative radiotherapy or chemoradiotherapy, usually due to advanced nodal diseases. This makes it difficult to analyze the additional oncological effect of TORS as a treatment.

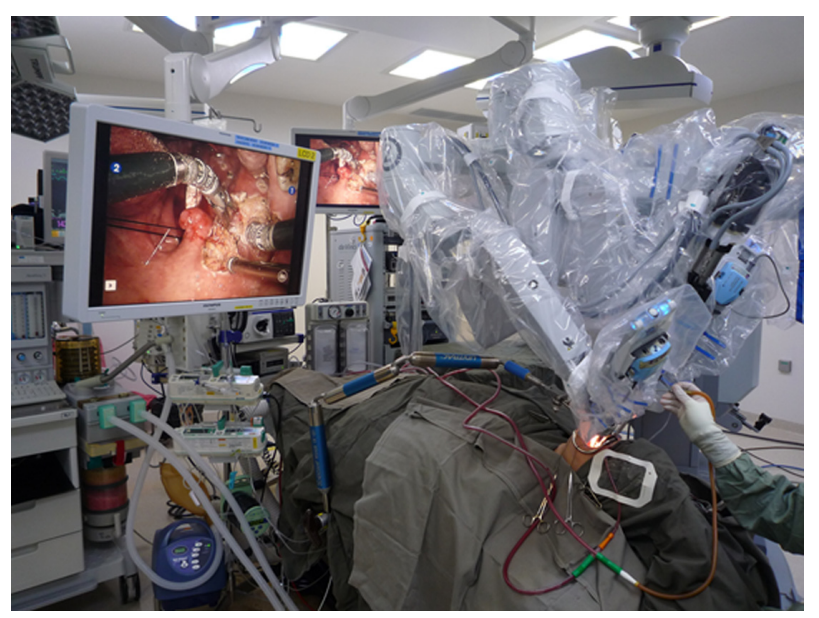

Figure 2 View of the patient and the robotic arms during robotic radical tonsillectomy.

Notes: The bedside surgical assistant's hand can be seen on the left of the photo, holding a suction. From the view of the video monitor, the tip of the suction can be seen inside the patient's oral cavity, evacuating smoke and blood.

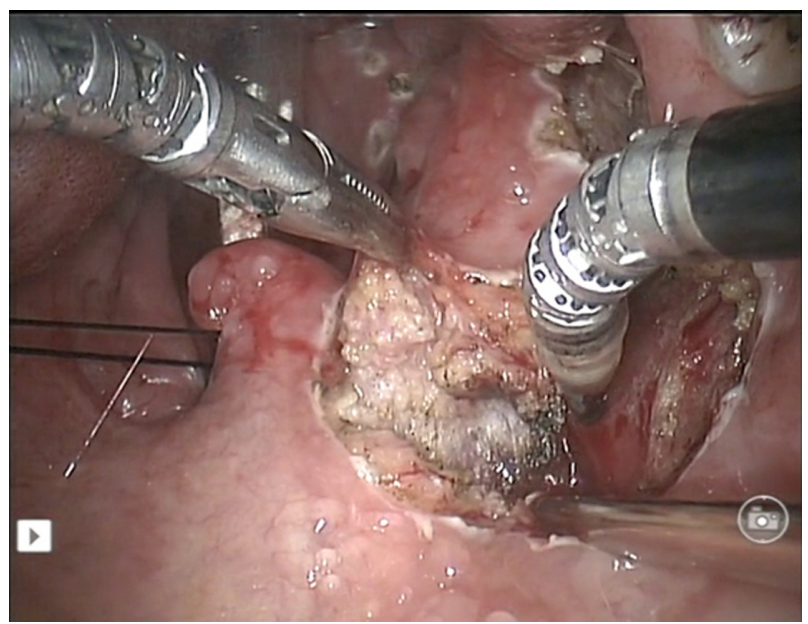

Figure 3 Screen capture of the robotic radical right tonsillectomy showing division of the superior constrictor muscles with the monopolar cautery diathermy spatula. Note: The other robotic arm was retracting the tonsil medially.

In the West, oropharyngeal cancer became the dominant type of cancer in the upper aerodigestive tract from the year $2000 .^{32}$ The majority of the oropharyngeal cancers harbor the human papilloma virus (HPV). ${ }^{33}$ Ang et al showed that HPV-related oropharyngeal cancer had a markedly improved survival after definitive treatment with chemoradiotherapy, and HPV was shown to be a strong and independent prognostic factor in oropharyngeal cancer. ${ }^{34}$ The 3 -year overall survival of the HPV-positive oropharyngeal cancer patients in Ang's cohort was $82.4 \%$, compared to $57.1 \%$ in the HPV-negative patients. Primary chemoradiotherapy was shown to affect the swallowing function of the patients to an extent that the patient could become feeding-tube dependent. ${ }^{33,35,36}$ Higher dose of

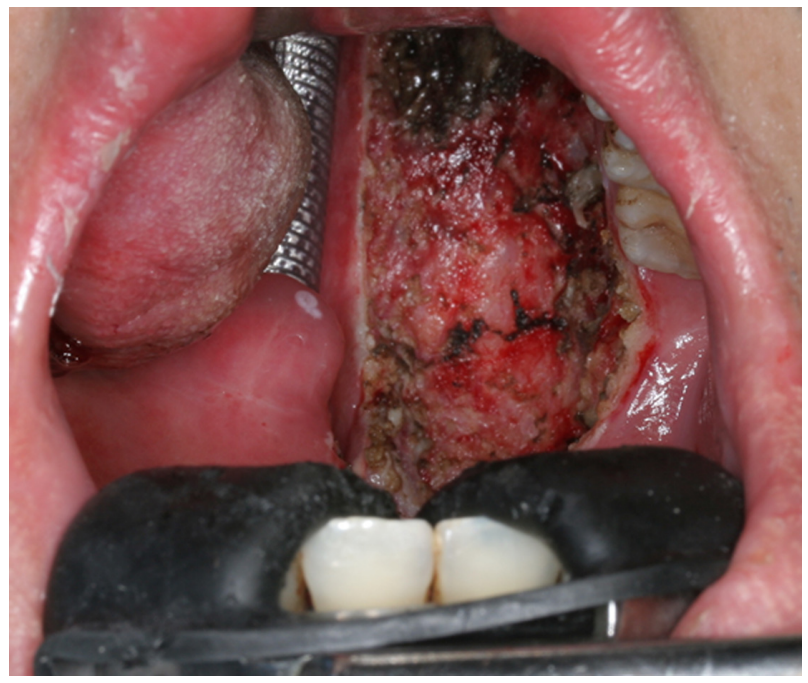

Figure $\mathbf{4}$ Immediate postoperative view of the surgical defect after robotic radial tonsillectomy.

Note: The defect would be left for healing with secondary intention. 


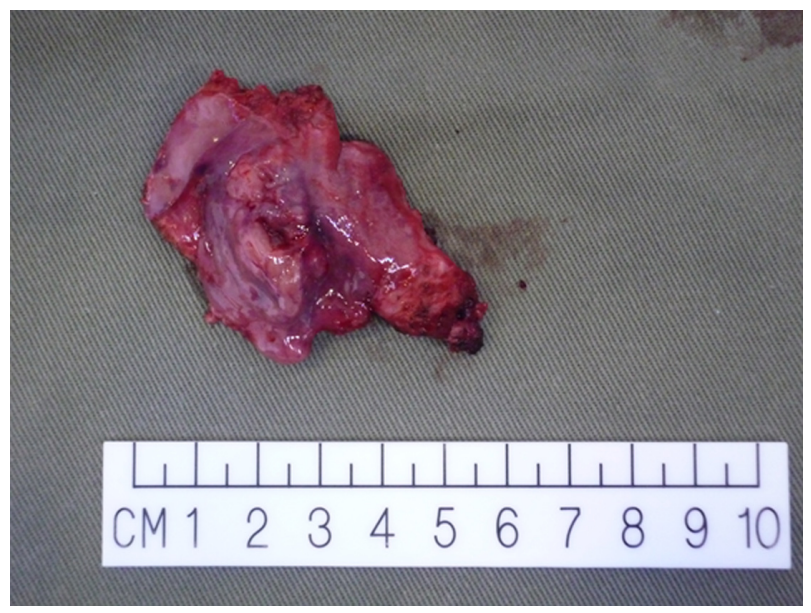

Figure 5 Photo of the radical tonsillectomy specimen.

radiotherapy and the addition of chemotherapy were shown to be a predictor of poor swallowing outcome..$^{34,36,37}$ As the survival of HPV-related oropharyngeal cancer is excellent, minimizing the long-term complications after treatment is necessary.

Advocates of TORS and primary surgical treatment argued that with primary surgery (TORS or other methods) to resect the local disease, and conservative neck dissection to control nodal disease, a significant portion of the patients can avoid high-dose radiotherapy or the addition of chemotherapy. This would in turn lead to a better long-term swallowing outcome. This is the advantage of incorporating TORS in the overall management of HPV-related oropharyngeal cancer, which is otherwise managed equally well with chemoradiotherapy in terms of survival outcome. Phase II randomized clinical trials are now ongoing or under planning to define the role of TORS in HPV-related and HPV-unrelated cancer of the oropharynx. ${ }^{38}$

There are fewer reports on the oncological efficacy of TORS in management of laryngeal cancer. ${ }^{14,39-42}$ The reported cohorts were of smaller size with a median of nine patients (range three to 18). All resections in the five cohorts achieved negative surgical margins. The initial outcomes were comparable to other modalities of treatment but there were no obvious advantages compared with other modalities of treatment like transoral laser microsurgery. A large multicenter report on the experience of TORS on 82 laryngeal cancers commented that only a small portion of patients would not be able to receive transoral laser microsurgery. ${ }^{17}$

To date, there are two reports on performing total laryngectomy with the da Vinci surgical robot, but both are small case series with no oncological outcome measurement. ${ }^{43,44}$
The advantage of robotic total laryngectomy is unknown at present.

Oncological results for TORS resection of hypopharyngeal cancers are also lacking. The largest series consisted of 23 patients with 16 T1-T2 tumors. ${ }^{45}$ Sixty-nine percent received adjuvant radiotherapy or chemoradiotherapy. The 3 -year overall survival was $89 \%$. Lörincz et al reported their experience in five patients using TORS to resect early (T1-T2) cancer of the pyriform fossa. ${ }^{46}$ They were able to achieve en bloc resection with at least $4 \mathrm{~mm}$ microscopic clear margins.

For TORS to be a successful minimally invasive surgery, the functional outcome should be as important as the oncological outcome. The majority of the functional outcome studies concentrated on swallowing function, as dysphagia is the main long-term morbidity of the alternative organ preservation treatments. The majority of the functional outcome reports are from TORS resection of oropharyngeal cancer. Hutcheson et al published a systematic review of the functional outcome after TORS for oropharyngeal cancer in $2014 .{ }^{47}$ The review included 12 trials with 441 patients. The conclusion was that patients who underwent TORS for oropharyngeal cancer had a lower gastrostomy utilization rate when compared to published benchmarks of radiotherapy or chemoradiotherapy cohorts. The subjective swallowing symptom scores for TORS patients were comparable to radiotherapy cohorts.

Richmon et al reported an analysis of 9,601 patients treated for oropharyngeal cancer with ablative surgery from 2008-2009 in the USA from the discharge data of the Nationwide Inpatient Sample. ${ }^{48}$ TORS was associated with shorter hospital stay (mean -1.5 days) and lower hospital related costs (mean -US\$4,285) after controlling for comorbidities, extent of surgery, and type of hospital. The study did not factor in the capital cost of the da Vinci surgical robot so it was difficult to extrapolate the real cost saving. The study also showed that patients who received TORS had a lower rate of gastrostomy and tracheostomy when compared with other surgical ablative procedures. Chung et al reported a nationwide analysis of clinical and cost outcome of TORS for oropharyngeal and oral tongue cases from 2008-2011 in the United States, and showed that TORS lateral oropharyngectomy and tongue base resection had clinical and cost benefits. There was no benefit with using the robot for oral tongue cancer. ${ }^{49}$

An emerging unique application of TORS in the management of head and neck malignancies is in the workup of the metastatic neck lymph node of unknown primary. Mehta 
et al first reported a case series of ten patients with metastatic neck lymph node of unknown primary who underwent TORS resection of base of tongue, and the primary cancer was identified in nine out of ten cases. ${ }^{50}$ Abuzeid et al reported a case of identifying the primary cancer in the base of tongue after TORS resection of base of tongue, and argued that the ability of TORS to identify the primary would spare patients from widefield irradiation if the primary was unable to be identified. ${ }^{51}$ Durmus et al showed in their cohort of 22 patients that the use of TORS tonsillectomy and base of tongue resection as part of the diagnostic and therapeutic procedure for unknown primary had excellent short-term quality of life outcome. ${ }^{52}$ Literature is still lacking in comparison of the incorporation of TORS versus standard workup of endoscopy and imaging in the workup of metastatic neck lymph node of unknown primary.

Apart from ablative surgery, the da Vinci surgical robot has also been employed in reconstruction of defects in the upper aerodigestive tract after resection of the primary tumor. Selber et al demonstrated in the dissection laboratory the feasibility of insetting a microvascular free flap in the oropharynx and microvascular anastomosis of the free flap in a cadaver. ${ }^{53}$ The advantage of the da Vinci surgical robot in flap inset was the ability to visualize and suture in the confined space of the base of tongue and oropharyngeal wall, while the disadvantage was the lack of tactile sensation in tying knots, requiring the surgeon to use vision to inspect the tightness of the knots. Since then, Selber et al and other groups have published their case series on robotic inset of free flaps. ${ }^{54-57}$

Despite the widespread use of TORS, robotic-assisted reconstructions of head and neck defects have not gained popularity. Apart from the additional cost incurred with using the robot, there are several reasons for the lack of popularity of robotic-assisted reconstructions in the head and neck region. Firstly, lesions resected with TORS are usually small and healing by primary closure, ${ }^{58}$ secondary intention, or local rotational mucosal flap would be adequate. Larger tumors are usually contraindicated for the TORS approach and are usually resected with traditional transcervical approaches with or without mandibulotomy. These defects would then be reconstructed in a traditional way. Secondly, the loss of tactile sensation may not be compensated by the superior visualization offered by the robot. Thirdly, the da Vinci surgical robot is still bulky and takes time to set up in preparation for use in flap insetting and microvascular anastomosis. Surgeons may not be willing to add this extra setup time in these already long operations, though Katz et al argued that the setup time for the robot is similar to the setup time of the microscope. ${ }^{59}$ Lastly, tremor of the surgeon during microvascular anastomosis is not an insurmountable obstacle and, with practice, most microvascular surgeons would be able to overcome hand tremor and perform microvascular surgery well. The benefit of tremor filtration offered by the da Vinci surgical robot may be marginal.

Apart from application in head and neck oncology, the da Vinci surgical robot has also been used for benign conditions of the head and neck region, most commonly for the resection of hypertrophic tongue base tissue in obstructive sleep apnea syndrome (OSAS). Principles and setup for TORS resection of base of tongue can be easily adapted to resect hypertrophied lingual tonsils in patients with OSAS. Several reports have been published in the literature on the use of the da Vinci surgical robot for resection of base of tongue and other redundant oropharyngeal soft tissue in OSAS. ${ }^{60-63}$ Further research is required to compare the efficacy and cost-benefit ratio in using the robot in comparison to other modalities like radiofrequency ablation and coblation lingual tonsillectomy. The risks associated with TORS procedures in OSAS are similar to other procedures in sleep apnea surgery, ${ }^{64}$ and special monitoring and nursing care need to be offered in the postoperative period to maximize patient safety. ${ }^{65}$

\section{Application of the da Vinci surgical robot in the nasopharynx and skull base}

Endoscopic endonasal surgery has gained significant advancements in the past 2 decades due to the improvement in surgical instruments and increased understanding of the endoscopic anatomy of the nasal cavities, paranasal sinuses, anterior skull base, and central skull base. Currently, a two surgeon four hands technique is a standard approach for endoscopic endonasal resection of skull base lesions. ${ }^{66}$ The next logical step in endoscopic endonasal surgery is the application of robotic surgery. ${ }^{67}$

The first preclinical trial on the use of the da Vinci surgical robot in the skull base was started in 2005 by the University of Pennsylvania group. ${ }^{68}$ They performed cadaveric dissections and showed the feasibility of using the robot to dissect in the parapharyngeal space and infratemporal fossa. The access to the parapharyngeal space and infratemporal fossa was through a transoral route with incision of the ipsilateral tonsillar pillar. They also noted that the absence of bone instruments would hamper resection of lesions involving the bony skull base. The group later described their case series of resecting benign tumors in the parapharyngeal space with the robot. ${ }^{6}$ 
Hanna et al described their approach to the anterior and central skull base using the da Vinci surgical robot on cadaveric dissection. ${ }^{70}$ Instead of employing a pure transoral approach, they created two "ports" on the anterior maxillary wall by two sublabial incisions and bilateral maxillary antrostomies (Caldwell-Luc approach). The middle meatal antrostomies of the maxillary sinuses were enlarged and the robotic arm could be advanced into the nasal cavity to reach the skull base. The endoscope was inserted into the nasal cavity through one of the nostrils. They demonstrated resection of lesions in the pituitary fossa, planum sphenoidale, nasopharynx, pterygopalatine fossa, and cribriform plate with the robotic arms. More importantly, they demonstrated the ability to perform watertight suturing of the anterior cranial fossa dura with the surgical robot. Some of the dissection was performed with prototype instruments that can remove bone in the skull base (E Hanna, personal communication, December 2013). These prototype instruments, unfortunately, have not been marketed and are not available for clinical use.

In 2010, Lee et al reported their investigation of various approaches to the skull base with the da Vinci surgical robot on cadaveric dissection. ${ }^{71}$ They commented that with transoral route and elevation of the soft palate, a 0 -degree lens would be adequate for visualization of lesions in the foramen magnum level. For lower and middle clivus, a 30-degree upwardlooking telescope setup would be required. Using a small $8.5 \mathrm{~mm} 30$-degree telescope, they could visualize the sella after removing the anterior sphenoid wall and sphenoid floor with conventional endoscopic drills. Unfortunately, clutter of robotic arms limited the range of movements of the robotic arms. In an effort to circumvent the clutter of robotic arms, the group had previously described placement of two ports through the submandibular soft tissue facing superiorly and allowed placing the two robotic arms outside the oral cavity angled superiorly. ${ }^{71,72} \mathrm{McCool}$ et al also described a similar setup in which the third robotic arm was placed in the port inserted above the hyoid bone into the oropharynx. ${ }^{73}$

Ozer and Waltonen described the first robotic nasopharyngectomy on a cadaver. ${ }^{74}$ To improve visualization of the nasopharynx, the soft palate was split in the midline and retracted laterally. They were able to resect the entire nasopharynx mucosa and both eustachian tube openings to expose the clivus and prevertebral fascia. The authors commented that the potential advantages of the robotic approach included no facial incisions and no osteotomies.

Dallan et al performed cadaveric dissection for robotic nasopharyngectomy using the submandibular ports and 0 -degree endoscope introduced through one nostril. ${ }^{75}$
They commented that this approach offered a better visualization of the roof of the nasopharynx and reduced conflicts of the robotic instruments and endoscope during the dissection.

Wei and Ho described the first clinical case of robotic nasopharyngectomy for recurrent nasopharyngeal carcinoma through a transoral approach. ${ }^{76}$ The approach was similar to the approach described by Ozer and Waltonen in their cadaveric dissection experiments. ${ }^{74}$ Since then, there have been further reports on the development of robotic nasopharyngectomy, including combined approach with endoscopic endonasal approach. ${ }^{77,78}$ Tsang et al reported the first clinical case series of 12 patients who underwent robotic nasopharyngectomy for small recurrent cancer. The early results were comparable with open surgery with less morbidity. ${ }^{79}$

O'Malley et al first described the use of TORS to resect parapharyngeal space tumors. ${ }^{69}$ Since then, there have been several large case series reporting the use of TORS for parapharyngeal tumors. Chan et al performed a systematic review of the use of TORS in resection of parapharyngeal tumors. ${ }^{80}$ They concluded that the advantages of using TORS compared to transcervical approach included the absence of a neck scar and absence of first bite syndrome after operation. Unfortunately, TORS approach needs an incision through the oropharyngeal mucosa and superior constrictor muscles and higher rate of capsule rupture during dissection. TORS for resection of parapharyngeal tumors may be advantageous in lesions situated medial to the carotid vessels. An external approach will require retraction of the carotid vessels for exposure and resection of the lesion, which has its associated risks. Vidhyadharan et al reported the use of TORS for resection of a second branchial arch cyst medial to the carotid vessels, and Ansarin et al reported a series of parapharyngeal space tumors in the post-styloid compartment resected with TORS approach. ${ }^{81,82}$

\section{Challenges and future developments}

The da Vinci surgical robot was not primarily designed to be deployed in the head and neck region. It is still too bulky to use in places like the anterior skull base. A new surgical robot (model: da Vinci Xi) with a smaller footprint and less chance of arms collision has already been launched in the market, but unfortunately has not been designed for transoral use and FDA approval for TORS has not been sought. Smaller robotic instruments with flexible or semiflexible arms are being designed. ${ }^{74,83}$ Flexible robots could be introduced through the oral cavity and turn upwards to operate in the nasopharynx, negating the requirement for a straight-line access for the current rigid robotic instruments. Richmon presented his 
Table I List of transoral robotic procedures mentioned in the review, excluding cadaveric experiments

Robotic radical tonsillectomy

Robotic tongue base resection

Robotic supraglottic laryngectomy

Robotic partial laryngectomy

Robotic hypopharyngectomy

Robotic total laryngectomy

Robotic-assisted transoral laser excision (oropharynx, larynx,

and hypopharynx)

Robotic-assisted insetting of flaps

Robotic microvascular anastomosis for free flaps

Robotic lingual tonsillectomy for sleep apnea

Robotic resection of parapharyngeal tumors

Robotic nasopharyngectomy

work on using the Flex ${ }^{\circledR}$ System robot to perform resection of the nasopharynx without transgressing the palate in a cadaver. $^{84}$

Lack of tactile feedback is a major criticism of robotic surgery and this is also true for TORS. Robotic surgeons currently substitute the tactile sensation mainly with visual cues to a certain extent, but visual cues could not entirely replace tactile sensation. VerroTouch is an early attempt to produce an external add-on to the da Vinci surgical robot to provide haptic feedback to the operating surgeon. ${ }^{75,85}$ The system is not required to be built into the current da Vinci robot, but instead is an after-market add-on. The sensors are placed on the wands of the robotic instruments just distal to the mounting points of the robotic arms. The vibration actuators are placed on the control handles of the console. As the robotic instruments slip or hit tissue, the sensor will sense the acceleration/deceleration. The electrical signals from the sensors will be filtered, amplified, and converted to vibrations in the actuators. The surgeon would be able to feel vibrations in the finger-tips. A small-scale survey of the advantage of the VerroTouch system has been completed by eleven robotic surgeons and the majority welcomed the addition of the haptic feedback, but none found it essential.

Development of robotic instruments for bone removal will definitely improve the application of the surgical robot in fields like spine surgery and anterior skull base surgery, where bone removal constitutes a significant proportion of the operation. Currently, traditional endoscopic drills and burs are used if bone removal is required during TORS for the skull base. Developing small-diameter articulated robotic drills and burs suitable for TORS is an engineering challenge. Alternatively, other approaches for bone removal may be more suitable for development into robotic instruments. Ultrasonic aspirators like Sonopet (Stryker, Kalamazoo,
MI, USA) can precisely remove bone and have been used in endoscopic endonasal skull base surgery. ${ }^{86}$ Currently, it is the small commercial demand that limits the development of robotic versions of these instruments.

The development of alternative energy-source instruments for hemostasis and their integration with robotic instruments will be a welcomed invention. Currently, TORS that use only monopolar and bipolar cautery are mostly being using for hemostasis. The current robotic ultrasonic dissector is too bulky and lacks articulation. An ultrasonic dissector would be a better instrument for dissection around areas prone to bleeding like venous plexus in the pterygoid plexus and parapharyngeal space.

The surgical navigation system can overcome the difficulty of identifying and avoiding injury of vital structures, especially the internal carotid artery in robotic nasopharyngectomy. The currently available optic or electromagnetic types of navigation systems in the market have difficulty in working with the surgical robot. A surgical robot with integrated navigation system is under active development and we should see clinical application in the near future. ${ }^{87}$

With the development of computer and imaging technology, augmented reality in surgical imaging is now available for clinical use. Augmented reality allows for the images of radiology scans to be overlaid on the endoscopic view during operation. This will allow better identification of pathologies and critical neurovascular structures buried deep underneath normal tissues. Integration of augmented reality with surgical robots has been under research for some time and clinical products should be available soon. ${ }^{88}$

\section{Conclusion}

In nearly a decade of development, TORS has emerged from a niche experimental surgery to mainstream management of early cancers in the oropharynx. It also has limited use in resecting cancers in the larynx and hypopharynx, and tumors in the skull base. TORS for benign conditions, especially tongue base resection for obstructive sleep apnea, has rapidly been adopted. TORS can be considered as the continuation of the paradigm shift first initiated by endoscopic surgery. With the advancement of technology, newer surgical robots, especially ones designed for application in TORS, will emerge soon. With the advent of these new technologies, surgeons must be prepared for another paradigm shift.

\section{Disclosure}

The author received a clinical research grant from Intuitive Surgical Inc. in 2012 for clinical research on robotic 
assisted nasopharyngectomy. The author reports no other conflicts of interest in this work.

\section{References}

1. Himpens J, Leman G, Cadiere GB. Telesurgical laparoscopic cholecystectomy. Surg Endosc. 1998;12(8):1091.

2. Cadière GB, Himpens J, Germay O, et al. Feasibility of robotic laparoscopic surgery: 146 cases. World J Surg. 2001;25(11):1467-1477.

3. Sammon J, Trinh QD, Menon M. Robotic radical prostatectomy: a critical analysis of surgical quality. Curr Opin Urol. 2011;21(3):195-199.

4. Lowery WJ, Leath CA 3rd, Robinson RD. Robotic surgery applications in the management of gynecologic malignancies. J Surg Oncol. 2012;105(5):481-487.

5. Trastulli S, Farinella E, Cirocchi R, et al. Robotic resection compared with laparoscopic rectal resection for cancer: systematic review and meta-analysis of short-term outcome. Colorectal Dis. 2012;14(4): e134-e156.

6. McLeod IK, Melder PC. Da Vinci robot-assisted excision of a vallecular cyst: a case report. Ear Nose Throat J. 2005;84(3):170-172.

7. Hockstein NG, Nolan JP, O'malley BW Jr, Woo YJ. Robotic microlaryngeal surgery: a technical feasibility study using the daVinci surgical robot and an airway mannequin. Laryngoscope. 2005;115(5): 780-785.

8. O’Malley BW Jr, Weinstein GS, Snyder W, Hockstein NG. Transoral robotic surgery (TORS) for base of tongue neoplasms. Laryngoscope. 2006;116(8): 1465-1472.

9. Hockstein NG, O'Malley BW Jr, Weinstein GS. Assessment of intraoperative safety in transoral robotic surgery. Laryngoscope. 2006;116(2): $165-168$.

10. Hockstein NG, Nolan JP, O’Malley BW Jr, Woo YJ. Robot-assisted pharyngeal and laryngeal microsurgery: results of robotic cadaver dissections. Laryngoscope. 2005;115(6):1003-1008.

11. O’Malley BW Jr, Weinstein GS, Hockstein NG. Transoral robotic surgery (TORS): glottic microsurgery in a canine model. $J$ Voice. 2006;20(2):263-268.

12. Weinstein GS, O'malley BW Jr, Hockstein NG. Transoral robotic surgery: supraglottic laryngectomy in a canine model. Laryngoscope. 2005;115(7):1315-1319.

13. Weinstein GS, O’Malley BW Jr, Snyder W, Sherman E, Quon H. Transoral robotic surgery: radical tonsillectomy. Arch Otolaryngol Head Neck Surg. 2007;133(12):1220-1226.

14. Weinstein GS, O'Malley BW Jr, Snyder W, Hockstein NG. Transoral robotic surgery: supraglottic partial laryngectomy. Ann Otol Rhinol Laryngol. 2007;116(1):19-23.

15. Park YM, Lee WJ, Lee JG, et al. Transoral robotic surgery (TORS) in laryngeal and hypopharyngeal cancer. $J$ Laparoendosc Adv Surg Tech A. 2009;19(3):361-368.

16. Genden EM, Desai S, Sung CK. Transoral robotic surgery for the management of head and neck cancer: a preliminary experience. Head Neck. 2009;31(3):283-289.

17. Vergez S, Lallemant B, Ceruse P, et al. Initial multi-institutional experience with transoral robotic surgery. Otolaryngol Head Neck Surg. 2012;147(3):475-481.

18. Weinstein GS, O’Malley BW Jr, Magnuson JS, et al. Transoral robotic surgery: a multicenter study to assess feasibility, safety, and surgical margins. Laryngoscope. 2012;122(8):1701-1707.

19. Desai SC, Sung CK, Jang DW, Genden EM. Transoral robotic surgery using a carbon dioxide flexible laser for tumors of the upper aerodigestive tract. Laryngoscope. 2008;118(12):2187-2189.

20. Remacle M, Matar N, Lawson G, Bachy V, Delos M, Nollevaux MC. Combining a new $\mathrm{CO} 2$ laser wave guide with transoral robotic surgery: a feasibility study on four patients with malignant tumors. Eur Arch Otorhinolaryngol. 2012;269(7):1833-1837.

21. Benazzo M, Canzi P, Occhini A. Transoral robotic surgery with laser for head and neck cancers: a feasibility study. ORL J Otorhinolaryngol Relat Spec. 2012;74(3):124-128.
22. Kucur C, Durmus K, Dziegielewski PT, Ozer E. Transoral robot- assisted carbon dioxide laser surgery for hypopharyngeal cancer. Head Neck. Epub September 15, 2014

23. White HN, Moore EJ, Rosenthal EL, et al. Transoral robotic-assisted surgery for head and neck squamous cell carcinoma: one- and 2-year survival analysis. Arch Otolaryngol Head Neck Surg. 2010;136(12):1248-1252.

24. Weinstein GS, O'Malley BW Jr, Cohen MA, Quon H. Transoral robotic surgery for advanced oropharyngeal carcinoma. Arch Otolaryngol Head Neck Surg. 2010;136(11):1079-1085.

25. Cohen M, Weinstein G, O’Malley B Jr, Feldman M, Quon H. Transoral robotic surgery and human papillomavirus status: Oncologic results. Head Neck. 2011;33(4):573-580.

26. Genden EM, Kotz T, Tong CC, et al. Transoral robotic resection and reconstruction for head and neck cancer. Laryngoscope. 2011;121(8): $1668-1674$

27. Hurtuk A, Agrawal A, Old M, Teknos TN, Ozer E. Outcomes of transoral robotic surgery: a preliminary clinical experience. Otolaryngol Head Neck Surg. 2011;145(2):248-253.

28. Cognetti DM, Luginbuhl AJ, Nguyen AL, Curry JM. Early adoption of transoral robotic surgical program: preliminary outcomes. Otolaryngol Head Neck Surg. 2012;147(3):482-488.

29. Lee SY, Park YM, Byeon HK, Choi EC, Kim SH. Comparison of oncologic and functional outcomes after transoral robotic lateral oropharyngectomy versus conventional surgery for T1 to T3 tonsillar cancer. Head Neck. 2014;36(8):1138-1145.

30. Park YM, Kim WS, Byeon HK, Lee SY, Kim SH. Oncological and functional outcomes of transoral robotic surgery for oropharyngeal cancer. Br J Oral Maxillofac Surg. 2013;51(5):408-412.

31. van Loon JW, Smeele LE, Hilgers FJ, van den Brekel MW. Outcome of transoral robotic surgery for stage I-II oropharyngeal cancer. Eur Arch Otorhinolaryngol. Epub March 8, 2014.

32. Marur S, D'Souza G, Westra WH, Forastiere AA. HPV-associated head and neck cancer: a virus-related cancer epidemic. Lancet Oncol. 2010;11(8):781-789.

33. Chaturvedi AK, Engels EA, Pfeiffer RM, et al. Human papillomavirus and rising oropharyngeal cancer incidence in the United States. J Clin Oncol. 2011;29(32):4294-4301.

34. Ang KK, Harris J, Wheeler R, et al. Human papillomavirus and survival of patients with oropharyngeal cancer. $N$ Engl J Med. 2010;363(1): 24-35.

35. Machtay M, Moughan J, Trotti A, et al. Factors associated with severe late toxicity after concurrent chemoradiation for locally advanced head and neck cancer: an RTOG analysis. J Clin Oncol. 2008;26(21): 3582-3589.

36. Bhayani MK, Hutcheson KA, Barringer DA, et al. Gastrostomy tube placement in patients with oropharyngeal carcinoma treated with radiotherapy or chemoradiotherapy: factors affecting placement and dependence. Head Neck. 2013;35(11):1634-1640.

37. Wilson JA, Carding PN, Patterson JM. Dysphagia after nonsurgical head and neck cancer treatment: patients' perspectives. Otolaryngol Head Neck Surg. 2011;145(5):767-771.

38. Adelstein DJ, Ridge JA, Brizel DM, et al. Transoral resection of pharyngeal cancer: summary of a National Cancer Institute Head and Neck Cancer Steering Committee Clinical Trials Planning Meeting, November 6-7, 2011, Arlington, Virginia. Head Neck. 2012;34(12):1681-1703.

39. Olsen SM, Moore EJ, Koch CA, Price DL, Kasperbauer JL, Olsen KD. Transoral robotic surgery for supraglottic squamous cell carcinoma. Am J Otolaryngol. 2012;33(4):379-384.

40. Mendelsohn AH, Remacle M, Van Der Vorst S, Bachy V, Lawson G. Outcomes following transoral robotic surgery: supraglottic laryngectomy. Laryngoscope. 2013;123(1):208-214.

41. Aubry K, Yachine M, Lerat J, Vivent M, Perez AF, Scomparin A. Transoral robotic surgery for the treatment of head and neck cancer of various localizations. Surg Innov. 2012;19(1):60-66.

42. Ozer E, Alvarez B, Kakarala K, Durmus K, Teknos TN, Carrau RL. Clinical outcomes of transoral robotic supraglottic laryngectomy. Head Neck. 2013;35(8):1158-1161.

43. Lawson G, Mendelsohn AH, Van Der Vorst S, Bachy V, Remacle M. Transoral robotic surgery total laryngectomy. Laryngoscope. 2013; 123(1):193-196. 
44. Smith RV, Schiff BA, Sarta C, Hans S, Brasnu D. Transoral robotic total laryngectomy. Laryngoscope. 2013;123(3):678-682.

45. Park YM, Kim WS, de Virgilio A, Lee SY, Seol JH, Kim SH. Transoral robotic surgery for hypopharyngeal squamous cell carcinoma: 3-year oncologic and functional analysis. Oral Oncol. 2012;48(6):560-566.

46. Lörincz BB, Busch C-J, Möckelmann N, Knecht R. Feasibility and safety of transoral robotic surgery (TORS) for early hypopharyngeal cancer: a subset analysis of the Hamburg University TORS-trial Eur Arch Otorhinolaryngol. Epub September 13, 2014.

47. Hutcheson KA, Holsinger FC, Kupferman ME, Lewin JS. Functional outcomes after TORS for oropharyngeal cancer: a systematic review. Eur Arch Otorhinolaryngol. Epub March 9, 2014.

48. Richmon JD, Quon H, Gourin CG. The effect of transoral robotic surgery on short-term outcomes and cost of care after oropharyngeal cancer surgery. Laryngoscope. 2014;124(1):165-171.

49. Chung TK, Rosenthal EL, Magnuson JS, Carroll WR. Transoral robotic surgery for oropharyngeal and tongue cancer in the United States. Laryngoscope. Epub August 5, 2014.

50. Mehta V, Johnson P, Tassler A, et al. A new paradigm for the diagnosis and management of unknown primary tumors of the head and neck: a role for transoral robotic surgery. Laryngoscope. 2013;123(1):146-151.

51. Abuzeid WM, Bradford CR, Divi V. Transoral robotic biopsy of the tongue base: A novel paradigm in the evaluation of unknown primary tumors of the head and neck. Head Neck. 2013;35(4):E126-E130.

52. Durmus K, Patwa HS, Gokozan HN, et al. Functional and quality of life outcomes of transoral robotic surgery for carcinoma of unknown primary. Laryngoscope. 2014;124(9):2089-2095.

53. Selber JC, Robb G, Serletti JM, Weinstein G, Weber R, Holsinger FC Transoral robotic free flap reconstruction of oropharyngeal defects: a preclinical investigation. Plast Reconstr Surg. 2010;125(3):896-900.

54. Mukhija VK, Sung CK, Desai SC, Wanna G, Genden EM. Transoral robotic assisted free flap reconstruction. Otolaryngol Head Neck Surg. 2009;140(1):124-125.

55. Selber JC. Transoral robotic reconstruction of oropharyngeal defects: a case series. Plast Reconstr Surg. 2010;126(6):1978-1987.

56. Garfein ES, Greaney PJ Jr, Easterlin B, Schiff B, Smith RV. Transoral robotic reconstructive surgery reconstruction of a tongue base defect with a radial forearm flap. Plast Reconstr Surg. 2011;127(6):2352-2354.

57. Bonawitz SC, Duvvuri U. Robot-assisted oropharyngeal reconstruction with free tissue transfer. J Reconstr Microsurg. 2012;28(7):485-490.

58. Nam IC, Park JO, Joo YH, Cho KJ, Kim MS. Role of primary closure after transoral robotic surgery for tonsillar cancer. Auris Nasus Larynx. Epub September 20, 2014

59. Katz RD, Taylor JA, Rosson GD, Brown PR, Singh NK. Robotics in plastic and reconstructive surgery: use of a telemanipulator slave robot to perform microvascular anastomoses. J Reconstr Microsurg. 2006;22(1):53-57.

60. Vicini C, Montevecchi F, Pang K, et al. Combined transoral robotic tongue base surgery and palate surgery in obstructive sleep apneahypopnea syndrome: expansion sphincter pharyngoplasty versus uvulopalatopharyngoplasty. Head Neck. 2014;36(1):77-83.

61. Lee JM, Weinstein GS, O'Malley BW Jr, Thaler ER. Transoral robotassisted lingual tonsillectomy and uvulopalatopharyngoplasty for obstructive sleep apnea. Ann Otol Rhinol Laryngol. 2012;121(10): 635-639.

62. Friedman M, Hamilton C, Samuelson CG, et al. Transoral robotic glossectomy for the treatment of obstructive sleep apnea-hypopnea syndrome. Otolaryngol Head Neck Surg. 2012;146(5):854-862.

63. Vicini C, Dallan I, Canzi P, et al. Transoral robotic surgery of the tongue base in obstructive sleep Apnea-Hypopnea syndrome: anatomic considerations and clinical experience. Head Neck. 2012;34(1):15-22.

64. Glazer TA, Hoff PT, Spector ME. Transoral Robotic Surgery for Obstructive Sleep Apnea: Perioperative Management and Postoperative Complications. JAMA Otolaryngol Head Neck Surg. Epub October 2, 2014.

65. Murray S. Nursing care for patients undergoing transoral robotic surgery. ORL Head Neck Nurs. 2009;27(1):8-12.

66. Snyderman CH, Carrau RL, Kassam AB, et al. Endoscopic skull base surgery: principles of endonasal oncological surgery. J Surg Oncol. 2008;97(8):658-664.
67. Castelnuovo P, Dallan I, Battaglia P, Bignami M. Endoscopic endonasal skull base surgery: past, present and future. Eur Arch Otorhinolaryngol. 2010;267(5):649-663.

68. O'Malley BW Jr, Weinstein GS. Robotic skull base surgery: preclinical investigations to human clinical application. Arch Otolaryngol Head Neck Surg. 2007;133(12):1215-1219.

69. O’Malley BW Jr, Quon H, Leonhardt FD, Chalian AA, Weinstein GS. Transoral robotic surgery for parapharyngeal space tumors. ORL $J$ Otorhinolaryngol Relat Spec. 2010;72(6):332-336.

70. Hanna EY, Holsinger C, DeMonte F, Kupferman M. Robotic endoscopic surgery of the skull base: a novel surgical approach. Arch Otolaryngol Head Neck Surg. 2007;133(12):1209-1214.

71. Lee JYK, O'Malley BW, Newman JG, et al. Transoral robotic surgery of the skull base: a cadaver and feasibility study. ORL J Otorhinolaryngol Relat Spec. 2010;72(4):181-187.

72. O'malley BW Jr, Weinstein GS. Robotic anterior and midline skull base surgery: preclinical investigations. Int J Radiat Oncol Biol Phys. 2007;69(2 Suppl):S125-S128.

73. McCool RR, Warren FM, Wiggins RH 3rd, Hunt JP. Robotic surgery of the infratemporal fossa utilizing novel suprahyoid port. Laryngoscope. 2010;120(9):1738-1743.

74. Ozer E, Waltonen J. Transoral robotic nasopharyngectomy: a novel approach for nasopharyngeal lesions. Laryngoscope. 2008;118(9): 1613-1616.

75. Dallan I, Castelnuovo P, Montevecchi F, et al. Combined transoral transnasal robotic-assisted nasopharyngectomy: a cadaveric feasibility study. Eur Arch Otorhinolaryngol. 2012;269(1):235-239.

76. Wei WI, Ho WK. Transoral robotic resection of recurrent nasopharyngeal carcinoma. Laryngoscope. 2010;120(10):2011-2014.

77. Yin Tsang RK, Ho WK, Wei WI. Combined transnasal endoscopic and transoral robotic resection of recurrent nasopharyngeal carcinoma. Head Neck. 2012;34(8):1190-1193.

78. Sreenath SB, Rawal RB, Zanation AM. The combined endonasal and transoral approach for the management of skull base and nasopharyngeal pathology: a case series. Neurosurg Focus. 2014;37(4):E2.

79. Tsang RK, To VS, Ho AC, Ho W-K, Chan JY, Wei WI. Early results of robotic assisted nasopharyngectomy for recurrent nasopharyngeal carcinoma. Head Neck. Epub March 7, 2014

80. Chan JYK, Tsang RK, Eisele DW, Richmon JD. Transoral Robotic Surgery (TORS) of the parapharyngeal space: A case series and systematic review. Head Neck. 2014

81. Vidhyadharan S, Krishnan S, King G, Morley A. Transoral robotic surgery for removal of a second branchial arch cyst: a case report. J Robot Surg. 2012;6(4):349-353.

82. Ansarin M, Tagliabue M, Chu F, Zorzi S, Proh M, Preda L. Transoral robotic surgery in retrostyloid parapharyngeal space schwannomas. Case Rep Otolaryngol. 2014;2014:296025.

83. Roppenecker DB, Meining A, Horst G, Ulbrich H, LuethTC. Interdisciplinary development of a single-port robot. Robotics and Biomimetics (ROBIO), 2012 IEEE International Conference on. 2012:612-617.

84. Richmon JD. Transoral palate-sparing nasopharyngectomy with the Flex ${ }^{\mathbb{R}}$ System: Preclinical study. Laryngoscope. Epub September 12, 2014.

85. Kuchenbecker KJ, Gewirtz J, McMahan W, et al. VerroTouch: HighFrequency Acceleration Feedback for Telerobotic Surgery. In: Haptics, Generating and Perceiving Tangible Sensations. Vol 6191. Lecture Notes in Computer Science. Berlin, Heidelberg: Springer Berlin Heidelberg; 2010:189-196.

86. Cappabianca P, Cavallo LM, Esposito I, Barakat M, Esposito F. Bone removal with a new ultrasonic bone curette during endoscopic endonasal approach to the sellar-suprasellar area: technical note. Neurosurgery. 2010;66(3 Suppl Operative):E118; discussion E118.

87. Xia T, Baird C, Jallo G, et al. An integrated system for planning, navigation and robotic assistance for skull base surgery. Int J Med Robot. 2008;4(4):321-330.

88. Volonté F, Pugin F, Bucher P, Sugimoto M, Ratib O, Morel P. Augmented reality and image overlay navigation with OsiriX in laparoscopic and robotic surgery: not only a matter of fashion. J Hepatobiliary Pancreat Sci. 2011;18(4):506-509. 
Robotic Surgery: Research and Reviews

Dovepress

\section{Publish your work in this journal}

Robotic Surgery: Research and Reviews is an international, peer reviewed, open access, online journal publishing original research, commentaries, reports, and reviews on the theory, use and application of robotics in surgical interventions. Articles on the use of supervisory-controlled robotic systems, telesurgical devices, and shared-control systems are

invited. The manuscript management system is completely online and includes a very quick and fair peer review system, which is all easy to use. Visit http://www.dovepress.com/testimonials.php to read real quotes from published authors.

Submit your manuscript here: http://www.dovepress.com/robotic-surgery-research-and-reviews-journal 